\title{
Establishment of a predictive nomogram and its validation for severe adenovirus pneumonia in children
}

\author{
Yang Shen^, Yao Zhou, Cuian Ma, Yuqiao Liu, Botao Wei \\ Department of Infectious Disease, Tianjin Children's Hospital/Tianjin University Children's Hospital, Tianjin, China \\ Contributions: (I) Conception and design: Y Shen, B Wei; (II) Administrative support: None; (III) Provision of study materials or patients: B Wei, C \\ Ma; (IV) Collection and assembly of data: Y Zhou, Y Liu; (V) Data analysis and interpretation: Y Shen, Y Zhou; (VI) Manuscript writing: All authors; \\ (VII) Final approval of manuscript: All authors. \\ Correspondence to: Botao Wei. Bachelor of Pediatrics, Chief Physician, Department of Infectious Disease, Tianjin Children's Hospital/Tianjin \\ University Children's Hospital, 238 Longyandao Road, Beichen District, Tianjin 300134, China. Email: weibotao2000@163.com.
}

\begin{abstract}
Background Severe adenovirus pneumonia (SAP) of children is prone to multi-system complications, has the high mortality rate and high incidence of sequelae. Severity prediction can facilitate an adequate individualized treatment plan. Our study try to develop and evaluate a predictive nomogram for children with SAP.

Methods: An observational study was designed and performed retrospectively. The data were categorized as training and validation datasets using the method of credible random split-sample (split ratio =0.7:0.3). The predictors were selected using Lasso (least absolute shrinkage and selection operator) logistic regression and the nomogram was developed. Nomogram discrimination was assessed using the receiver operating characteristic (ROC) curve, and the prediction accuracy was evaluated using a calibration curve. The nomogram was also evaluated for clinical effectiveness by the decision curve analysis (DCA). A P value of $<0.05$ was deemed statistically significant.
\end{abstract}

Results: The identified predictors were fever duration, and interleukin-6 and CD4+ T cells and were assembled into the nomogram. The nomogram exhibited good discrimination with area under ROC curve in training dataset (0.79, 95\% CI: 0.60-0.92) and test dataset (0.76, 95\% CI: 0.63-0.87). The nomogram seems to be useful clinically as per DCA.

Conclusions: A nomogram with a potentially effective application was developed to facilitate individualized prediction for SAP in children.

Keywords: Severe adenovirus pneumonia (SAP); nomogram; Lasso regression; validation; prognostic model

Submitted Mar 22, 2021. Accepted for publication May 21, 2021.

doi: 10.21037/apm-21-675

View this article at: https://dx.doi.org/10.21037/apm-21-675

\section{Introduction}

The common cause of diseases such as gastroenteritis, respiratory infections, and conjunctivitis are human adenoviruses (HAdV) are, especially in children under 5 years of age (1), and is responsible for nearly $5-10 \%$ of acute lower respiratory tract infections (ALRTIs) (2). Clinically, about one third of children with adenovirus pneumonia progress to severe disease (3). SAP is prone to multi-system complications, has a high mortality rate, and has a high incidence of sequelae, which places a heavy burden on society and families. Therefore, it is helpful to improve the prognosis of children with the early identification of children at risk of transition to severe symptoms when adenovirus pneumonia occurs, timely strengthening

\footnotetext{
^ ORCID: 0000-0002-9394-1322.
} 
monitoring and effective treatment. And it is also important for the allocation of medical resources. Importantly, the patient must be informed about the complete procedure and the possible associated complications. Severity prediction can facilitate an adequate individualized treatment plan to prevent morbidity.

Several studies have assessed the risk factor for SAP (4-6). Although many studies have proposed predictors for the prediction of SAP in children (7-9), none of them has been universally accepted for individualized prediction based on scientific evidence and is seldom associated with the visual prediction model. Nomogram has been considered lately to be an efficient visualization tool (10). It is particularly reliable and useful in the predictive model visualization (11) and for quantifying risks. Nomograms specifically score individual risk by numerical determining the probability of an event that is designed as per an individual patient's profile. The use of nomograms is facilitated by graphical interfaces that are convenient for generating these estimates during clinical scenarios for informed decision-making (12). However, there has been only little on the use of nomogram for accurate estimation of adenovirus pneumonia severity in children.

In earlier studies, results were derived from a commonly used, traditional method, the stepwise regression model. However, for small samples, it is less sensitive and can worsen the prediction error worse in a few cases $(13,14)$. Although it has played an important role, their studies may be more reasonable if they had considered this situation. Recently, machine learning has received much attention to identify biomarkers and predict outcomes in population studies. Lasso (least absolute shrinkage and selection operator) regression is a powerful tool according to the bias-variance tradeoff in situations of variable election (15). However, not much attention has been paid to Lasso in terms of a predictive nomogram for determining $\mathrm{SAP}$ in children.

Therefore, there is a need for studies on individualized prediction of adenovirus pneumonia severity by using Lasso penalized regression in patients. This study mainly focused on the development and validation of a nomogram for predicting the severity of adenovirus pneumonia in children. The investigators hypothesized that it is possible to validate and visualize a more reliable and accurate prediction model by a nomogram.

We present the following article in accordance with the STROBE reporting checklist (available at https://dx.doi. org/10.21037/apm-21-675).

\section{Methods}

\section{Design of the study and patient selection}

We retrospectively conducted an observational, crosssectional study in a clinical setting. The study sample included patients who were presented to the Tianjin Children's Hospital (January 2019 to December 2019), which conforms to the guideline for diagnosis and treatment of adenovirus pneumonia in children (2019 version) (16) and has only evidence of adenovirus infection at the beginning of admission the basis for the diagnosis of adenovirus pneumonia. The study was conducted in accordance with the Declaration of Helsinki (as revised in 2013). The study was approved by Ethics Board of Tianjin Children's Hospital (No. L2021-02) and individual consent for this retrospective analysis was waived.

Inclusion criteria and the pathogenic diagnosis is based on any of the following: (I) nasopharyngeal swab virus antigen positive; (II) nasopharyngeal swab adenovirus nucleic acid positive; (III) serum adenovirus-specific IgM antibody positive; (IV) detect adenovirus nucleic acid sequences in bronchoalveolar lavage fluid (BALF) and metagenomics next generation sequencing (mNGS).

Exclusion criteria were the following: (I) the course of illness exceeds 2 weeks when admitted to the hospital; (II) nosocomial infection; (III) children with severe allergic constitution; (IV) deaths or automatic discharge during hospitalization.

In this study, the Type 2a scheme mentioned by TRIPOD (Transparent Reporting of a Multivariable Prediction Model for Individual Prognosis or Diagnosis) (10) was used to build the model. One sixty-eight patients were arbitrarily categorized into training and testing datasets comprised respectively of, 122 and 46 patients according to the ratio of $7: 3$. The predictive nomogram was developed using the training dataset and the performance was evaluated using the testing dataset (Figure 1).

\section{Study predictors}

Some demographic characteristics, clinical data and laboratory tests of children were selected as variables in this study. Demographic characteristics, including gender, age, birth weight, premature delivery and basic disease conditions (for instance: chronic pulmonary problems, cardiac disease, neurologic diseases, severe malnutrition, anatomic malformations, hematologic malignancies or immunodeficiency) were selected as variables in this study, 


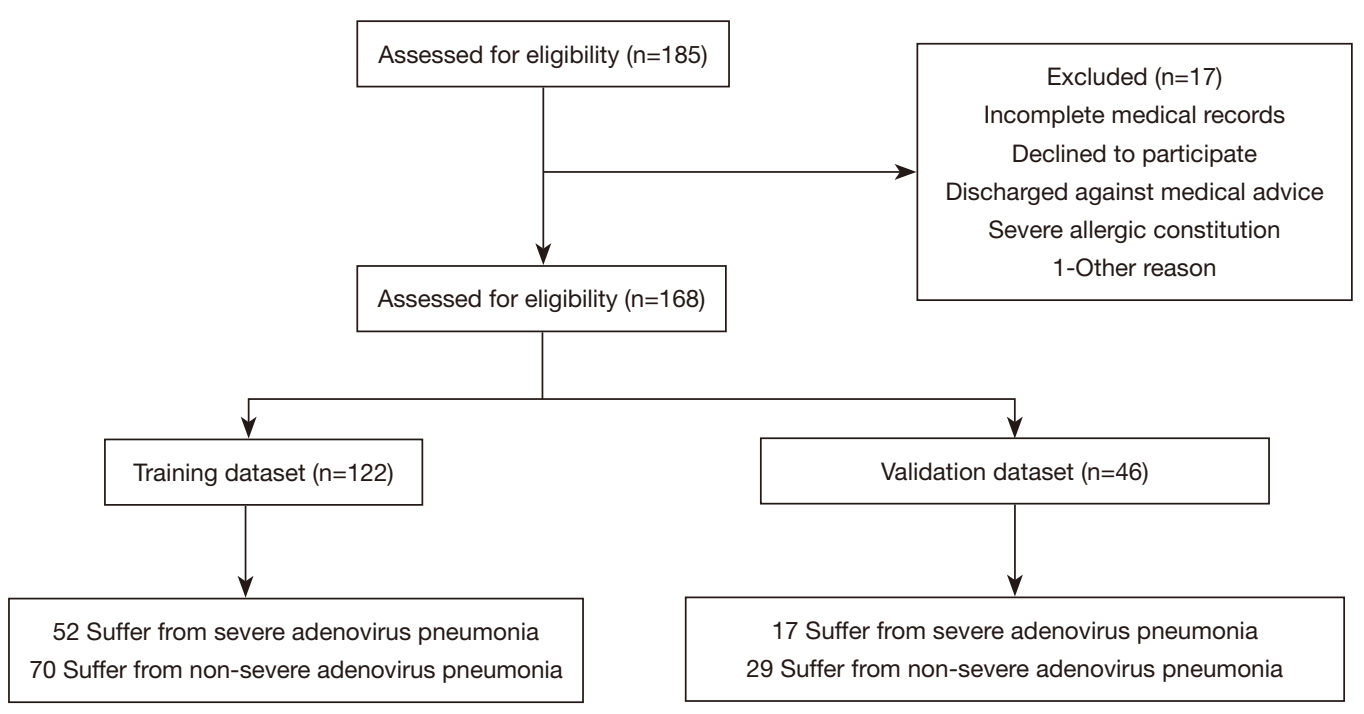

Figure 1 The numbers of enrolled participants and results of the training and testing datasets.

as well as clinical data and laboratory tests of children. Peak fever, duration of fever, mixing with other pathogenic infections contained within the clinical data. Fever was defined as an axillary temperature of $\geq 37.3{ }^{\circ} \mathrm{C}$. The peak fever selects the highest body temperature in the whole disease heat range. The definition of the duration of fever was the time from the onset of fever to the date of to be hospitalized. Etiological examination of blood and respiratory secretions were completed within 24 hours of admission, including influenza type $\mathrm{A}$ and $\mathrm{B}$, respiratory syncytial virus, Coxsackie group B virus, adenovirus, Mycoplasma pneumoniae, Chlamydia pneumoniae and germiculture. Our study chose the patients have only evidence of adenovirus infection at the beginning. We repeated the above pathogenic examination for patients who have not improved after 1 week of hospitalization or who have fever again after improvement. Mixed infection is defined as the evidence of other pathogens than adenovirus at this moment, it can be one or more. Our study selected the interception of maximum abnormalities from laboratory indicators during hospitalization, which including the quantity of leucocytes [white blood cell (WBC)], percentage of neutrophils and lymphocyte $(\mathrm{N} \%, \mathrm{~L} \%), \mathrm{C}$ reactive protein (CRP), procalcitonin (PCT), lactate dehydrogenase (LDH), interleukin-6 (IL-6), lactic acid (La), and the ratio of CD4+, CD8+, CD19+, CD16+CD56+, CD4+/CD8+ which obtained by flow cytology. The variables of training dataset and validation dataset were defined in Table 1. Data were collected using a standardized form for each operation for which the designated recorder was present. In order to avoid potential bias, all data was clearly defined exposure prior to study, and collectors were received standardized training.

\section{Primary outcomes}

The SAP was the outcome variable in this study. Diagnostic criteria for severe pneumonia which conforms to the diagnostic criteria of community acquired pneumonia (CAP) (17), there is any of the following: (I) poor general situation; (II) denial of food or dehydration; (III) consciousness disorders; (IV) faster breathing frequency (infant $>70 \cdot \mathrm{min}^{-1}$; older children $>50 \cdot \mathrm{min}^{-1}$ ); (V) cyanosis; (VI) dyspnoea (moaning, three concave signs, nasal wing fan); (VII) lung infiltration (multi-pulmonary leaves or $\geq 2 / 3$ of lungs); (VIII) pleural effusion; (IX) percutaneous oxygen saturation $\leq 0.92$, (X) extrapulmonary issues (cerebral abscess, meningitis, pericarditis, osteomyelitis, endocarditis, arthritis, hemolytic uremia syndrome, sepsis, etc.).

All the patient was dichotomized into the severity of adenovirus pneumonia in children as severe and non-severe according to criteria above, which was indicated by 1 and 0 , respectively.

\section{Selection of the predictor}

For the most practical predictive features in the training 
Table 1 Summary of study variables grouped by training and testing datasets*

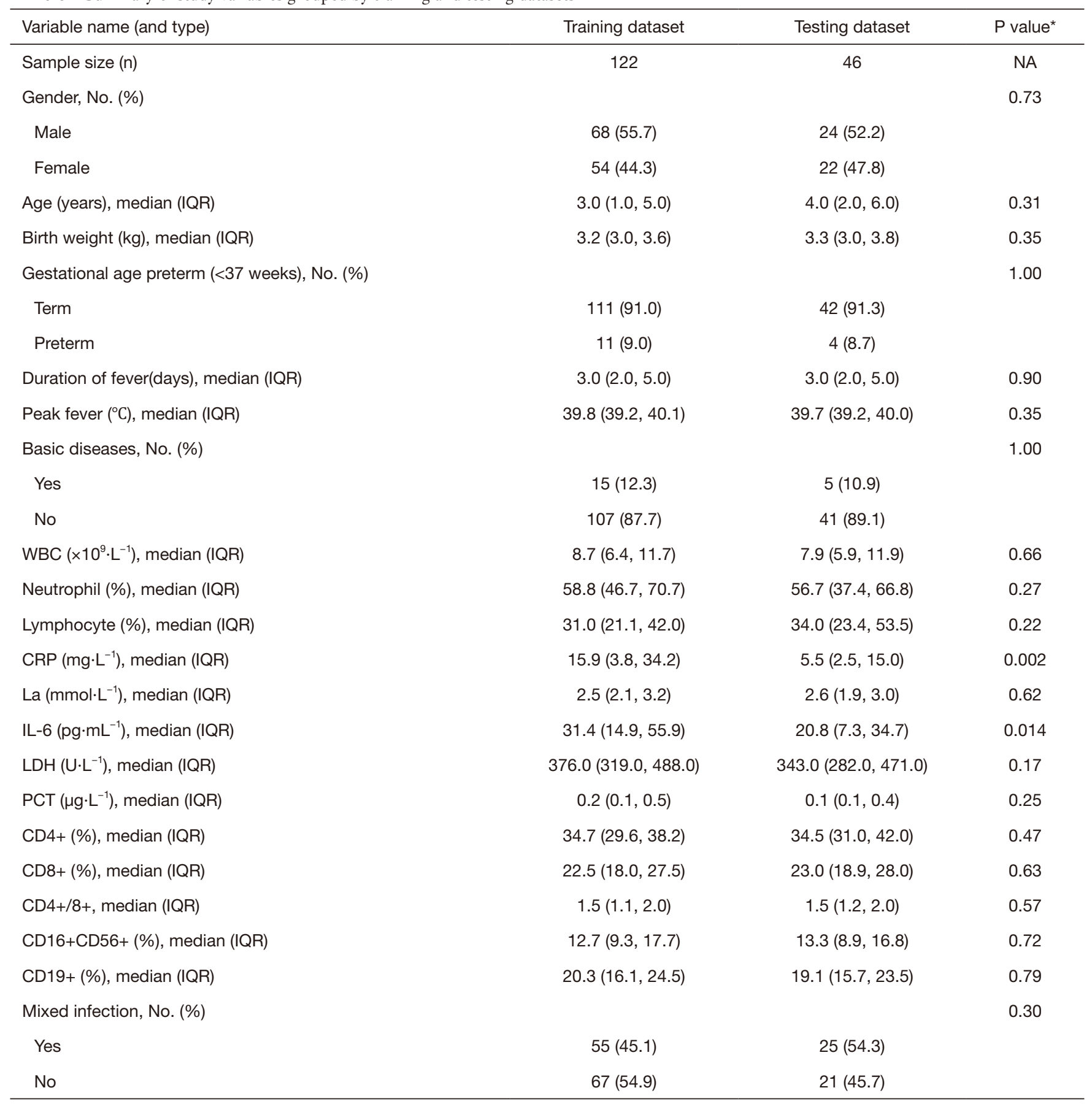

Data presented as median (IQR) or numbers, with percentages in parentheses. *, Mann-Whitney $U$ test was used for the continuous variables and Fisher's exact test was used for categorical variables specified in the exact argument. NA, not applicable; IQR, interquartile range; WBC, white blood cell; CRP, C reactive protein; PCT, procalcitonin; LDH, lactate dehydrogenase; La, lactic acid; IL-6, interleukin-6. 
dataset, we used the Lasso algorithm $(15,18)$. The binary logistic regression model involves the replacement of the residual sum of squares by the negative log-likelihood. The estimated regression parameters are not affected if the $\lambda$ is smaller, but a few coefficients may shrink to zero as the $\lambda$ increases $(15,18)$. We then chose the $\lambda$ with the smallest cross-validation error. Finally, the model was re-fit using the selected $\lambda$ and all available observations. The majority of the covariate coefficients decreased to zero and the other non-zero coefficients are chosen using Lasso.

\section{Statistical analysis}

Analyses of statistical data were carried out using R V4.0.1 (R Foundation for Statistical Computing) and STATA 16.0 for Windows (StataCorp Texas, USA). Bivariate analysis was carried out using the Fisher's exact chi-squared test for categorical and Mann-Whitney $U$ test for continuous variables. The retrospective nature of the study predetermines the sample size. For Lasso feature selection, the "glmnet" R package was applied (19). to develop a predictive model, we used the binary logistic regression model. Then, using the "rms" R package, a nomogram was built. Discrimination of the model was assessed through the ROC (receiver operating characteristic) curve. To determine the clinical use of the nomogram, the decision curve was analyzed in the validation dataset by the quantification of net benefits at different threshold probabilities (20). Each statistical test was twosided, and $\mathrm{P}$ values of $<0.05$ were deemed significant.

\section{Results}

\section{Patients' clinical characteristics}

The hospital enrolled 92 boys and 76 girls in ages ranging from 2 months to 14 years old. which included nonsevere $(n=99)$ and severe $(n=69)$ adenovirus pneumonia. The characteristics of patients in the training dataset and validation dataset are mentioned in Table 1 . The two datasets did not differ significantly in the SAP $(\mathrm{P}=0.29)$. In the training and validation datasets, the percent of patients with severe adenovirus pneumonia (SAP) was $42.6 \%$ and $37.0 \%$, respectively. Bivariate analyses of study variables versus severity for training dataset were displayed in Table 2 .

\section{Nomogram development}

Lasso regression analyses identified CD4+ T cells, IL-6, and duration of fever as predictors (Figure 2). The model incorporating the above-mentioned independent predictors was evolved and defined as the nomogram (Figure 3).

\section{Nomogram validation}

\section{Discrimination}

The AUC (area under the roc curve) is plotted in Figure 4. This nomogram exhibited excellent discrimination with an AUC of 0.79 (95\% CI: $0.60-0.92)$ in the training dataset (Figure 4).

\section{Clinical use}

Figure 5 presents the decision curve analysis (DCA) for the nomogram. DCA was performed on our prediction model to estimate the net benefit to the patients. The decision curve shows an obvious net benefit of the nomogram model for nearly all threshold probabilities, particularly the threshold probabilities between 20-60\% (Figure 5). However, in the case of less than $20 \%$ threshold probability, the nomogram exhibited a net benefit comparable to predicted positive results for all participants.

\section{Discussion}

HADV has a history of more than 70 years since its successful separation. It has been found that there are 7 subgenes from A to G, more than 103 types, and the population is generally susceptible (21). HADV is a common pathogen causing community-acquired pneumonia in children. A respiratory aetiological analysis of community-acquired pneumonia in hospitalized children suggests that the positive rate of adenovirus infection is about $3.5-9.4 \%$ (22). A report said that $53.9 \%$ of the 104 children with HADV7 infection were diagnosed with severe pneumonia (23). When SAP progressed with multiple organ dysfunction syndrome (MODS) in children, the mortality is higher over $50 \%$ (24). In this study, we developed and examined a nomogram to evaluate adenovirus pneumonia severity in children. It can efficiently discriminate individualized predictions and ease individualized treatment. This will aid in enhanced decision-making by the clinicians and patients to gain more net benefits.

The analysis results indicate that the duration of fever, CD4+ T cells and IL-6 levels are reliable predictors of SAP in children. The patients in the SAP group experienced a fever for a longer duration after the onset of symptom and admission, which was found on one study on the clinical 
Table 2 Bivariate analyses of study variables versus severity for training dataset

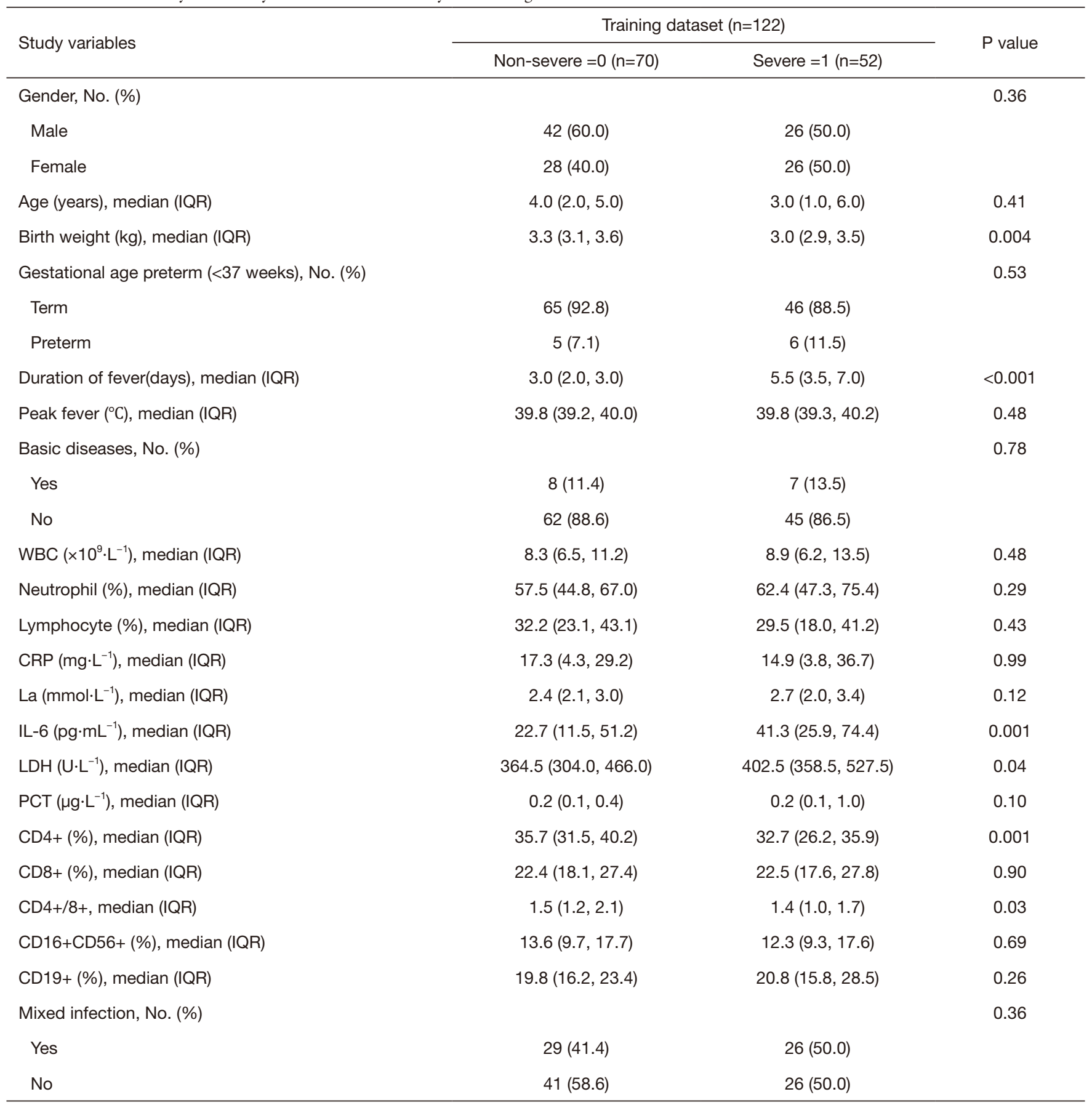

Mann-Whitney $U$ test was used for the continuous variables and Fisher's exact test was used for categorical variables specified in the exact argument. IQR, interquartile range; WBC, white blood cell; N\%, percentage of neutrophils; CRP, C reactive protein; PCT, procalcitonin; LDH, lactate dehydrogenase; La, lactic acid; IL-6, interleukin-6. 
A

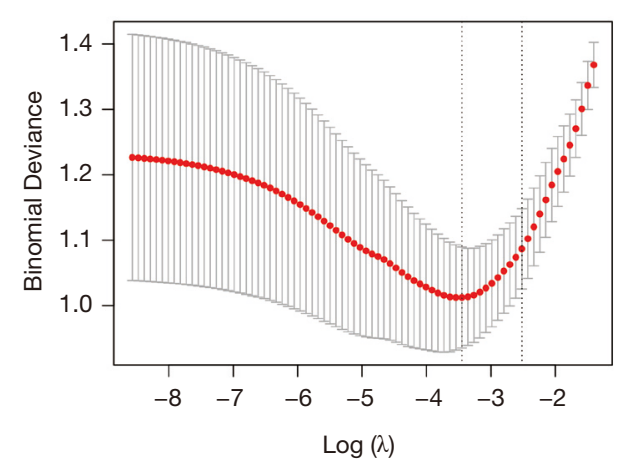

B

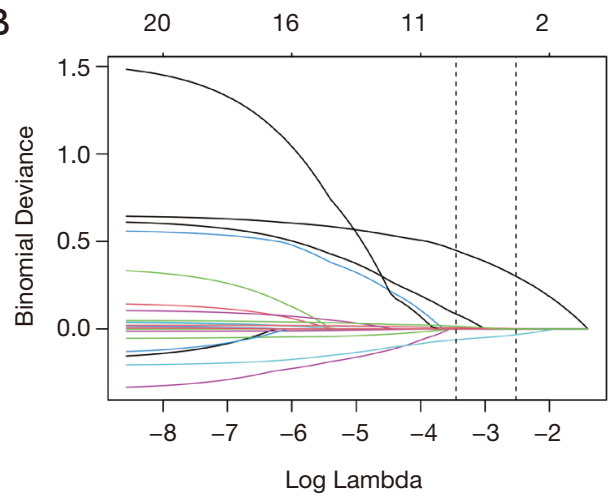

Figure 2 Selection of predictor using the LASSO (least absolute shrinkage and selection operator) binary logistic regression model. (A) Identification of lambda $(\lambda)$, or the optimal penalization coefficient in the Lasso model using minimum criterion and 10-fold crossvalidation. (B) Lasso coefficient profiles of the features. A vertical line was drawn at the selected value using 10-fold cross-validation, and optimal values were obtained by applying the 1 standard error of the minimum criteria (the 1-se criteria) and minimum criteria.

Points

duration of fever

IL.6

CD4

Total Points
1020

$\begin{array}{lllllll}40 & 50 & 60 & 70 & 80 & 90 & 100\end{array}$

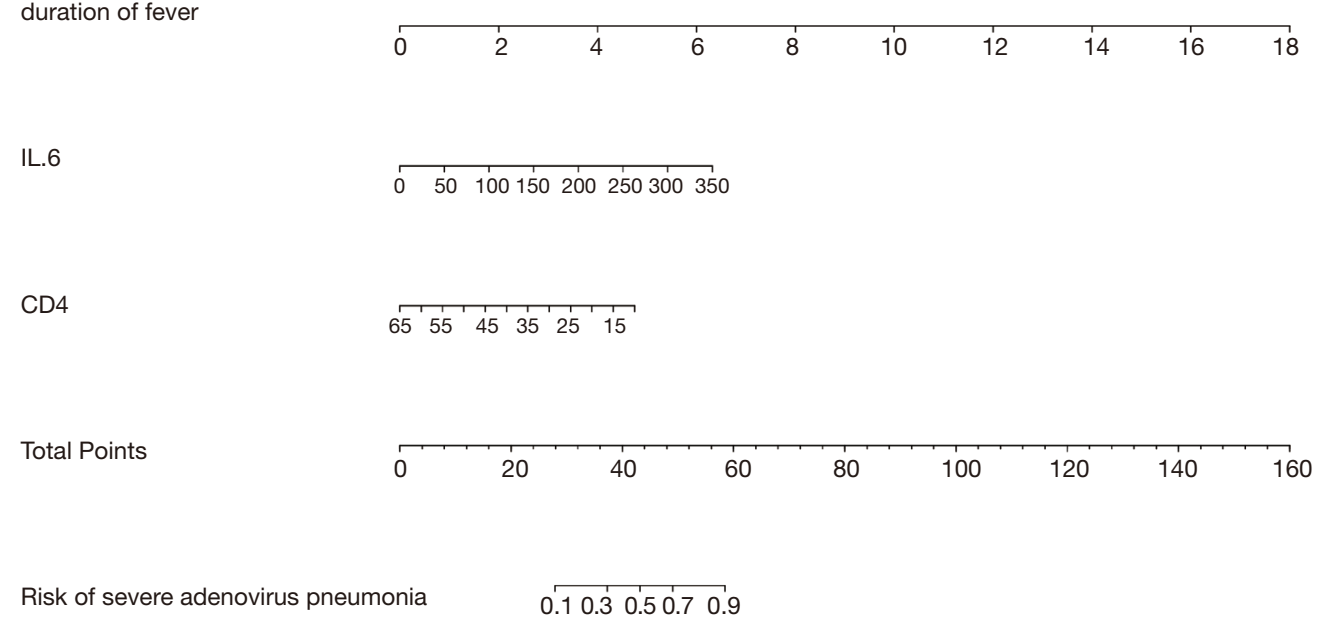

Figure 3 The use of nomogram to determine the probability of severity of adenovirus pneumonia in children. A nomogram for severity was designed and assimilated with the predictors. The predictor points were found on the uppermost point scale that matched with each patient variable and was added. The total points extrapolated to the bottom scale show the percent probability of severity.

characteristics of adenovirus pneumonia (25). Another report confirmed the fever duration associated with SAP was prolonged relative to that of mild-moderate pneumonia (26). A recently research showed the time of fever before admission is a high-risk factor for children with adenovirus pneumonia, and the duration of fever after admission, too, is a factor causing high-risk of critical illness and death (8). It suggested that long fever is an effective predictor of whether SAP is at risk, which is consistent with our study.

The innate immune response has a crucial role in the host response to HAdV infections (27). CD4+ T cells are able to recognize HADV capsid protein and cause the positive immune response (28). A CD4+ $\mathrm{T}$ cell is divided into Th1 and Th2 based on the different cytokine spectrum, while Th1 is able to cause cyto-immune effects through secreted cytokines to enhance resistance to infection. 


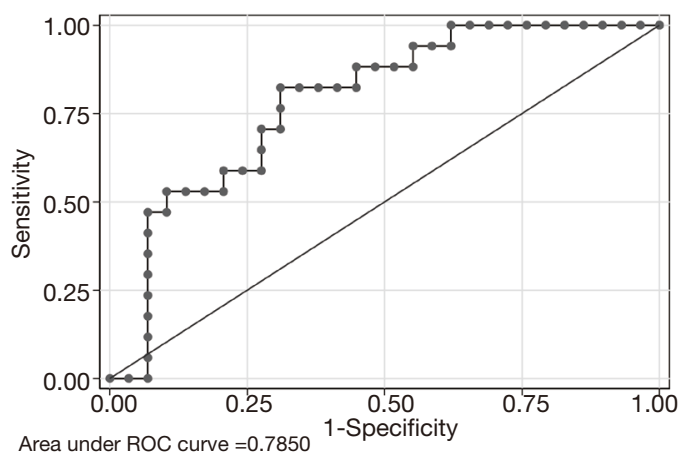

Figure 4 Nomogram ROC curves in the training dataset. The nomogram exhibited excellent power of discrimination with AUC (95\% confidence interval) of 0.785 (95\% CI: 0.60-0.92) in the training dataset. ROC, receiver operating characteristic; AUC, area under ROC curve.

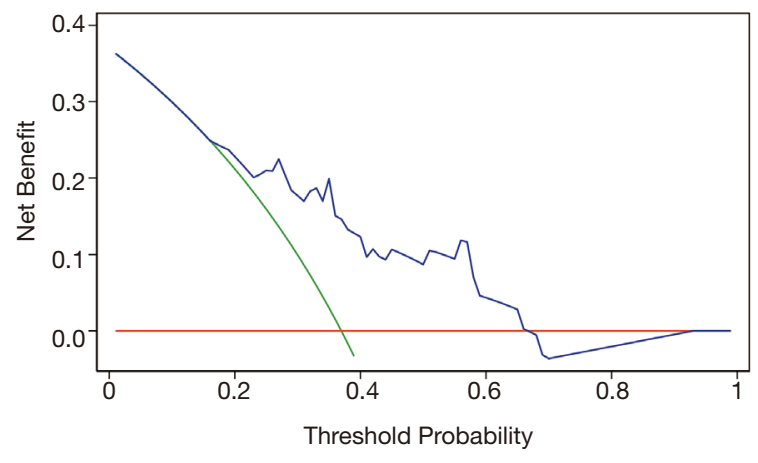

- Net Benefit: Treat All
Net Benefit: Nomogram

Figure 5 Decision curve analysis of the nomogram. The y-axis indicates the net benefit. The nomogram is represented by blueline. The green line indicates the presumption that all patients had severe adenovirus pneumonia. The pink line indicates the presumption that no patients had severe adenovirus pneumonia. The net benefit was estimated by subtracting the proportion of patients who tested false positive from the true positive proportion, weighted by the relative harm of not getting treated in comparison to the adverse outcomes of an unrequired treatment (19). The decision curve indicates that when a patient or doctor threshold probability is $>20 \%$ and $<60 \%$, using the nomogram to predict the severity of adenovirus pneumonia, it adds a greater advantage than the treat-all-patients severity or the treat-none severity.
Th2 performs humoral immunity by secreting cytokines to assist $\mathrm{B}$ cell activation and producing antibodies. An early animal experiment suggested intravenous injection of large doses of adenovirus caused a significant decrease in the number CD4+ $\mathrm{T}$ cells at 1 day post-challenge in non-human primates (29). Recently study confirmed that when SAP occurs, the number of CD4+ T cells decreases significantly, resulting in cellular immunosuppression in the body (30). The results indicates that the decrease of CD4 T cells in follow-up is a risk factor of SAP, and confirmed the correctness of the previous report.

IL-6 is both able to promote B cell to produce antibodies and to promote liver synthesis of a variety of acute timephase protein (31). It is found by Sun that the levels of IL-6 increased significantly in children who were in severe adenovirus infection (32). To those children (such as Kawasaki disease) who infected adenovirus pneumonia, their IL-6 level is significantly higher than other types of viral pneumonia (33). The studies have confirmed that IL-6 is associated with inflammatory reactions in lungs, and its overexpression may be associated with immune damage to the lungs (34). So it can be used as an indicator for predicting SAP (35), which is consistent with our study. Adenovirus-induced inflammation storms are decisive for prognosis of disease, which is very similar to the clinical characteristics of adult novel coronavirus pneumonia.

In Wu's study, low lymphocyte count and high serum $\mathrm{LDH}$ could be used to predict the severity of respiratory infection due to adenovirus in children (36). Gray's multivariable risk factor modeling for adenovirus disease severity founded that age and chronic disease increased the risk of severe disease (37). Cheng's report showed prematurity and basic neurologic diseases as risk factors for severe infections due to HAdV (9). However, these variables are not built in our model. The main reason for the difference with the previous research is that the observational population research is not fixed, and the representativeness of data, the range of variables, the size of sample and the characteristics of population will have different model results. Moreover, it is crucial to explain the results by clinical significance. In this study, a strict statistical method is used to screen out variables, and the clinical significance of this model is also clear. 
In this study, DCA was applied to assess its clinical usefulness $(38,39)$. This new method indicates clinical consequences based on the threshold probability or Pt. By using nomogram in the current study, the decision curve reveals that it is more advantageous to assess the severity of adenovirus pneumonia than the treat-all-patients severe or the treat-none severe cases under the circumstance of $>10 \%$ threshold probability of a doctor to a patient (Figure 5). An obvious net benefit of the nomogram model is observed for nearly all threshold probabilities, particularly in the range of $20-60 \%$ (Figure 5). However, in the case of $<10 \%$ threshold probability, the net benefit of the nomogram was comparable to the predicted positive results for all participants.

Our study has a few limitations too. First, our study was conducted at a single-center with relatively small sample size and may have some effect on the results to some level. The findings in this study need to be corroborated in intervention studies in the future. Further, for generalization, the nomogram must be confirmed in large sample sizes of multi-center studies. Second, our study did not include radiographic imaging. Preoperative radiographic imaging examination in future research may be necessary. Third, because of laboratory conditions, specific typing of adenovirus was not done, which may have led to measurement bias. Despite its limitations, this nomogram effectively performed as a tool for prediction and patient counseling.

To conclude, we developed a nomogram and verified it in predicting the SAP, and can be effortlessly used for individualized prediction and treatment of the SAP in children.

\section{Acknowledgments}

We would like to thank Editideas (www.editideas.cn) for English language editing.

Funding: None.

\section{Footnote}

Reporting Checklist: The authors have completed the STROBE reporting checklist. Available at https://dx.doi. org/10.21037/apm-21-675

Data Sharing Statement: Available at https://dx.doi. org/10.21037/apm-21-675
Peer Review File: Available at https://dx.doi.org/10.21037/ apm-21-675

Conflicts of Interest: All authors have completed the ICMJE uniform disclosure form (available at https://dx.doi. org/10.21037/apm-21-675). The authors have no conflicts of interest to declare.

Ethical Statement: The authors are accountable for all aspects of the work in ensuring that questions related to the accuracy or integrity of any part of the work are appropriately investigated and resolved. The study was conducted in accordance with the Declaration of Helsinki (as revised in 2013). The study was approved by Ethics Board of Tianjin Children's Hospital (No. L2021-02) and individual consent for this retrospective analysis was waived.

Open Access Statement: This is an Open Access article distributed in accordance with the Creative Commons Attribution-NonCommercial-NoDerivs 4.0 International License (CC BY-NC-ND 4.0), which permits the noncommercial replication and distribution of the article with the strict proviso that no changes or edits are made and the original work is properly cited (including links to both the formal publication through the relevant DOI and the license). See: https://creativecommons.org/licenses/by-nc-nd/4.0/.

\section{References}

1. Castro-Rodriguez JA, Daszenies C, Garcia M, et al. Adenovirus pneumonia in infants and factors for developing bronchiolitis obliterans: a 5-year follow-up. Pediatr Pulmonol 2006;41:947-53.

2. Tabain I, Ljubin-Sternak S, Cepin-Bogović J, et al. Adenovirus respiratory infections in hospitalized children: clinical findings in relation to species and serotypes. Pediatr Infect Dis J 2012;31:680-4.

3. Lu MP, Ma LY, Zheng Q, et al. Clinical characteristics of adenovirus associated lower respiratory tract infection in children. World J Pediatr 2013;9:346-9.

4. Zhong L, Lin J, Dai J. Risk factors for the development of bronchiolitis obliterans in children with severe adenovirus pneumonia: A retrospective study with dose-response analysis. J Med Virol 2020;92:3093-9.

5. Du F, Huang Y, Shu C, et al. Mixed infection and risk factors in children with severe adenovirus pneumonia. Zhongguo Dang Dai Er Ke Za Zhi 2013;15:375-8. 
6. Rajkumar V, Chiang CS, Low JM, et al.Risk Factors for Severe Adenovirus Infection in Children during an Outbreak in Singapore. Ann Acad Med Singap 2015;44:50-9.

7. Zampoli M, Mukuddem-Sablay Z. Adenovirus-associated pneumonia in South African children: Presentation, clinical course and outcome. S Afr Med J.2017;107:123-6.

8. Huang H, Chen Y, Ma LY, et al. Analysis of the clinical features and the risk factors of severe adenovirus pneumonia in children. Zhonghua Er Ke Za Zhi 2021;59:14-9.

9. Cheng JL, Peng CC, Chiu NC, et al. Risk factor analysis and molecular epidemiology of respiratory adenovirus infections among children in northern Taiwan, 2009-2013. J Microbiol Immunol Infect 2017;50:418-26.

10. Moons KG, Altman DG, Reitsma JB, et al. Transparent Reporting of a multivariable prediction model for Individual Prognosis or Diagnosis (TRIPOD): explanation and elaboration. Ann Intern Med 2015;162:W1-73.

11. Van Zee KJ, Manasseh DM, Bevilacqua JL, et al. A nomogram for predicting the likelihood of additional nodal metastases in breast cancer patients with a positive sentinel node biopsy. Ann Surg Oncol 2003;10:1140-51.

12. Iasonos A, Schrag D, Raj GV, et al. How to build and interpret a nomogram for cancer prognosis. J Clin Oncol 2008;26:1364-70.

13. Hurvich CM, Tsai CL. The impact of model selection on inference in linear regression. Am Stat 1990;44:214-7.

14. Roecker EB. Prediction error and its estimation for subset selected models. Technometrics 1991;33:459-68.

15. Tibshirani R. Regression shrinkage and selection via the lasso. J R Stat Soc B 1996;58:267-88.

16. National Health Commission of the People's Republic of China, State Administration of Traditional Chinese Medicine. Guideline for diagnosis and treatment of adenovirus pneumonia in children (2019 version). Chin J of Clin Infect Dis 2019;12:161-6.

17. Subspecialty Group of Respiratory Diseases, The Society of Pediatrics; Chinese Medical Association The Editorial Board, Chinese Journal of Pediatrics. Guidelines for management of community acquired pneumonia in children (the revised edition of 2013) (II). Zhonghua Er Ke Za Zhi 2013;51:856-62.

18. Tibshirani R. The lasso method for variable selection in the Cox model. Stat Med 1997;16:385-95.

19. Friedman J, Hastie T, Tibshirani R. Regularization paths for generalized linear models via coordinate descent. J Stat Softw 2010;33:1-22.

20. Vickers AJ, Elkin EB. Decision curve analysis: a novel method for evaluating prediction models. Med Decis Making 2006;26:565-74.

21. Gu J, Su QQ, Zuo TT, et al. Adenovirus diseases: a systematic review and meta-analysis of 228 case reports. Infection 2021;49:1-13.

22. Barnadas C, Schmidt DJ, Fischer TK, et al. Molecular epidemiology of human adenovirus infections in Denmark, 2011-2016. J Clin Virol 2018;104:16-22.

23. Wo Y, Lu QB, Huang DD, et al. Epidemical features of HAdV-3 and HAdV-7 in pediatric pneumonia in Chongqing, China. Arch Virol 2015;160:633-8.

24. Chowdhury F, Shahid ASMSB, Ghosh PK, et al. Viral etiology of pneumonia among severely malnourished under-five children in an urban hospital, Bangladesh. PLoS One 2020;15:e0228329.

25. Yoo H, Oh J, Park C. Characteristics of fever and response to antipyretic therapy in military personnel with adenovirus-positive community-acquired pneumonia. Mil Med Res 2020;7:6.

26. Park JY, Kim BJ, Lee EJ, et al. Clinical Features and Courses of Adenovirus Pneumonia in Healthy Young Adults during an Outbreak among Korean Military Personnel. PLoS One 2017;12:e0170592.

27. Hendrickx R, Stichling N, Koelen J, et al. Innate immunity to adenovirus. Hum Gene Ther 2014;25:265-84.

28. Onion D, Crompton LJ, Milligan DW, et al. The CD4+ T-cell response to adenovirus is focused against conserved residues within the hexon protein. J Gen Virol. 2007;88:2417-25.

29. Schnell MA, Zhang Y, Tazelaar J, et al. Activation of innate immunity in nonhuman primates following intraportal administration of adenoviral vectors. Mol Ther 2001;3:708-22.

30. Mistchenko AS, Koch ER, Kajon AE, et al. Lymphocyte subsets and cytokines in adenoviral infection in children. Acta Paediatr 1998;87:933-9.

31. Norris CA, He M, Kang LI, et al. Synthesis of IL-6 by hepatocytes is a normal response to common hepatic stimuli. PLoS One 2014;9:e96053.

32. Sun J, Xiao Y, Zhang M, et al. Serum Inflammatory Markers in Patients with Adenovirus Respiratory Infection. Med Sci Monit 2018;24:3848-55.

33. Kawasaki Y, Hosoya M, Katayose M, et al. Correlation between serum interleukin 6 and C-reactive protein concentrations in patients with adenoviral respiratory infection. Pediatr Infect Dis J 2002;21:370-4.

34. Ye Q, Wang B, Mao J. The pathogenesis and treatment of the 'Cytokine Storm' in COVID-19. J Infect 2020;80:607-13. 
35. Patel JA, Nair S, Ochoa EE, et al. Interleukin-6-174 and tumor necrosis factor $\alpha-308$ polymorphisms enhance cytokine production by human macrophages exposed to respiratory viruses. J Interferon Cytokine Res 2010;30:917-21.

36. Wu PQ, Zeng SQ, Yin GQ, et al. Clinical manifestations and risk factors of adenovirus respiratory infection in hospitalized children in Guangzhou, China during the 2011-2014 period. Medicine (Baltimore) 2020;99:e18584.

37. Gray GC, McCarthy T, Lebeck MG, et al. Genotype prevalence and risk factors for severe clinical adenovirus

Cite this article as: Shen Y, Zhou Y, Ma C, Liu Y, Wei B. Establishment of a predictive nomogram and its validation for severe adenovirus pneumonia in children. Ann Palliat Med 2021;10(7):7194-7204. doi: 10.21037/apm-21-675 infection, United States 2004-2006. Clin Infect Dis 2007;45:1120-31.

38. Steyerberg EW, Vickers AJ, Cook NR, et al. Assessing the performance of prediction models: a framework for traditional and novel measures. Epidemiology 2010;21:128-38.

39. Vickers AJ, Cronin AM, Elkin EB, et al. Extensions to decision curve analysis, a novel method for evaluating diagnostic tests, prediction models and molecular markers. BMC Med Inform Decis Mak 2008;8:53. 